\title{
DETECCIÓN DE UN NUEVO CAMPAMENTO ROMANO EN LAS INMEDIACIONES DE MÉRIDA MEDIANTE TECNOLOGÍA LiDAR
}

\section{INTRODUCCIÓN}

En las últimas décadas, el crecimiento exponencial de trabajos interesados en la Arqueología Militar de época romana ha permitido cubrir un importante hueco de la historiografía arqueológica peninsular, destacándose el análisis del proceso de conquista e implantación del poder romano entre los ss. II-I a.n. e. Esta línea de trabajo se ha centrado, especialmente, en el estudio de los campamentos legionarios (castra), entre cuyos problemas destaca el poco conocimiento disponible sobre su relación con eventos históricos concretos. Así pues, en esta breve noticia abordamos la contextualización de una serie de estructuras localizadas mediante LiDAR en la rivera de Lácara, afluente del río Guadiana en las inmediaciones de Mérida, a las cuales atribuimos un carácter militar, especialmente una de ellas, de tipo castra, con el fin de aportar nuevos datos que ayuden a obtener una mejor comprensión de la implantación romana en la cuenca media del Guadiana. Este trabajo se realiza, además, en un momento en el que la detección de estructuras militares romanas mediante esta técnica se ha popularizado y parece abrir nuevas vías de análisis (CostaGarcía y Casal 2015; Costa-García et al. 2016).

\section{METODOLOGÍA DE TRATAMIENTO DE LOS DATOS LIDAR E IDENTIFICACIÓN E INTER- PRETACIÓN DE LAS ESTRUCTURAS}

El caso de estudio que motiva la redacción de este trabajo se detectó por medio de la cobertura sistemática mediante tecnología LiDAR del territorio actual de Extremadura, de la que este trabajo es uno de los primeros avances. La información técnica es la habitual para los datos del PNOA: una densidad de puntos media es de 0.5 por $\mathrm{m}^{2}$ y precisión vertical inferior a $6 \mathrm{~cm}$ (error cuadrático medio). Los archivos LiDAR de una amplia región se procesaron en un entorno de supercomputación, que ha reducido en un $95 \%$ el tiempo invertido respecto a un ordenador convencional, para lo que empleamos un código informático propio (Cerrillo-Cuenca 2017). El MDT (modelo digital del terreno) obtenido, con $1 \mathrm{~m}$ de resolución, refleja fielmente las anomalías topográficas del terreno susceptibles de corresponder a estructuras arqueológicas, sobre él se han realizado el reconocimiento e interpretación de las estructuras que presentamos en este trabajo. Para facilitar la interpretación visual de los datos se crearon, automáticamente, sombreados analíticos de los 


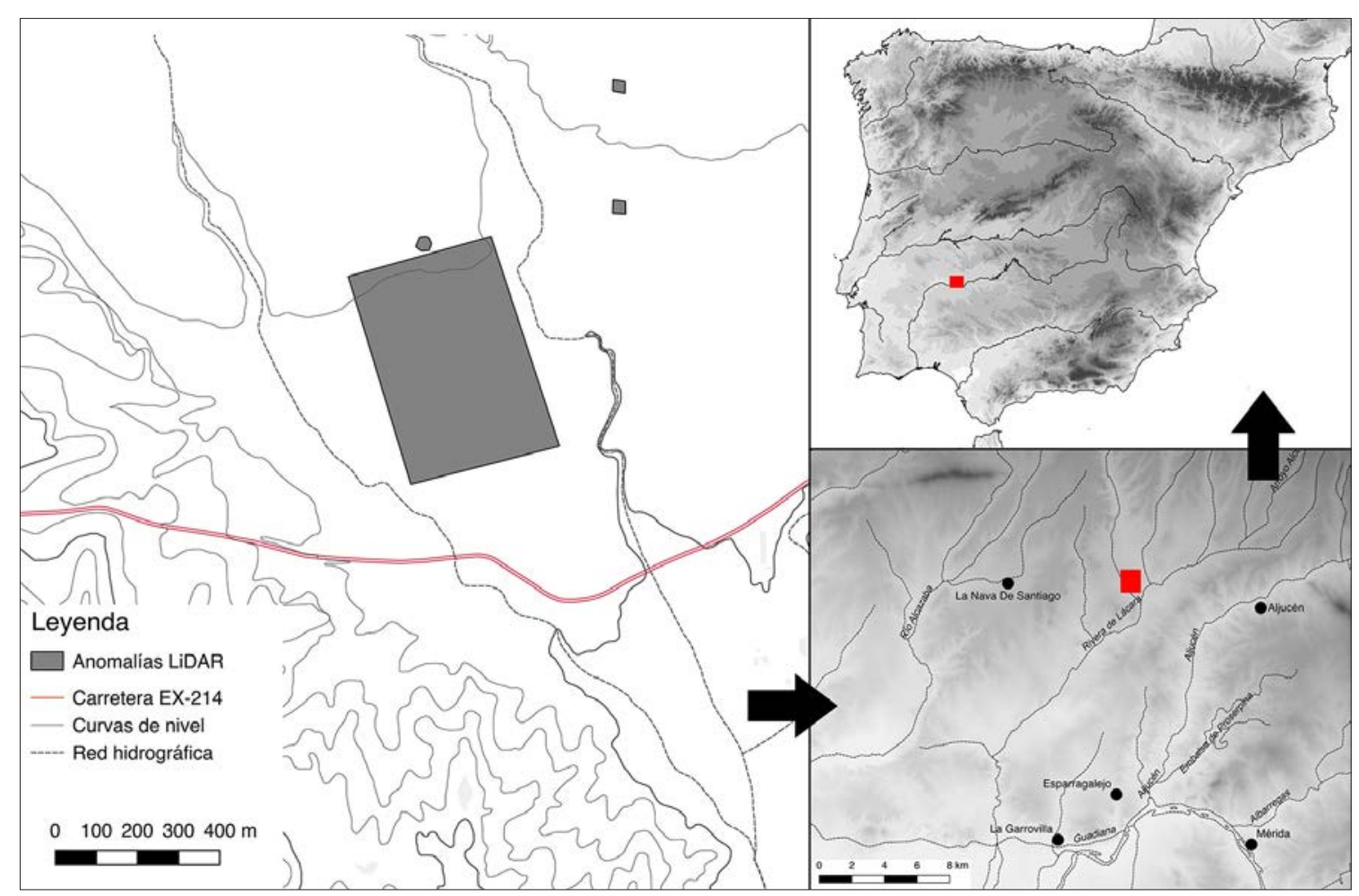

Fig. 1: Localización de las estructuras documentadas en la rivera de Lácara (Mérida, Badajoz).

MDT (Štular et al. 2012), además de emplear otras técnicas de visualización alternativas (Hesse 2010), una de ellas, muy útil en este trabajo, la laplaciana de la gaussiana (Zhan et al. 2011).

Las estructuras documentadas se inscriben entre la rivera de Lácara y el arroyo Zamorilla, en el término municipal de Mérida (fig. 1). El paisaje actual presenta una cobertura de encinar disperso, lo que dificulta su identificación mediante series históricas de fotografías aérea, y únicamente mediante la inspección sistemática de sombreados analíticos del MDT ha resultado posible su identificación (fig. 2). La principal anomalía identificada presenta forma rectangular con unas dimensiones aproximadas de $525 \mathrm{~m}$ para el lado mayor y $365 \mathrm{~m}$ para el lado menor, lo que supone una superficie aproximada de unas 17 ha. El sitio está delimitado por un foso y, al menos en el lado $\mathrm{N}$, conserva aún un alzado que ha dejado su huella en la topografía, probablemente los restos de una estructura defensiva. Los fosos están levemente indicados en la esquina SE, bien porque se han colmatado sin dejar una huella aparente en la topografía o bien porque las tareas agrícolas han acabado por desmontarlos. La medición de su anchura es compleja ya que se realiza a partir de sombreados analíticos, pero podemos observar que en el foso $\mathrm{N}$ la profundidad máxima documentada es de unos $0,5 \mathrm{~m}$, mientras que en la zona $\mathrm{SE}$ apenas sobrepasa los 0,2 $\mathrm{m}$. En el exterior de esta zona se aprecia, también, una depresión circular de $15 \mathrm{~m}$ de radio y de 2,5 m de profundidad, de explicación incierta. Por otro lado, en la zona interior sólo se distingue una somera elevación en la zona central, que es de difícil interpretación. Esta morfología no deja lugar a dudas y permite su identificación como un castra (Sabugo 2007: 22), ya que además presenta un sistema defensivo similar a otros ejemplos próximos como Cáceres el Viejo, con un tamaño superior al aquí presentado. Pero además, tanto por su posición entre dos corrientes como por su tamaño, recuerda a otros castra meseteños también analizados con técnicas similares a las descritas, como es el caso del campamento de los Llanos (CostaGarcía y Casal 2015: 147). La carencia de datos no nos permite por ahora avanzar en el carácter del campamento: estacional (castra aestiva) o permanente (castra hiberna o stativa) (Morillo 2008: 74-77). 


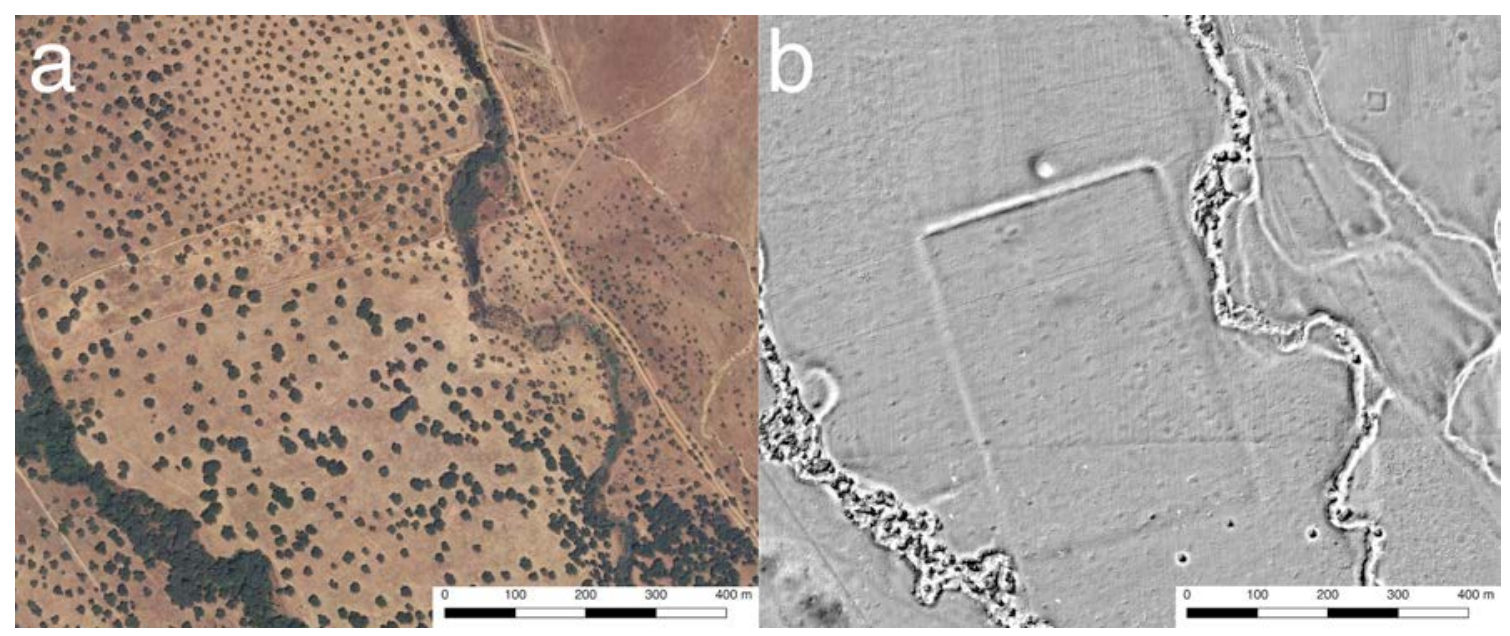

Fig. 2: Comparativa entre una ortoimagen aérea y los datos del LiDAR del castra estudiado. A - Ortoimagen del Plan Nacional de Ortofografía Aérea (PNOA) del año 2013. B - Datos LiDAR (PNOA), la imagen combina un sombreado analítico del terreno y los resultados del cálculo de la laplaciana de la gaussiana para el MDT.

Finalmente, al E del arroyo Zamorilla se aprecian dos anomalías de planta cuadrangular. Ambas estructuras presentas lados regulares de $22 \mathrm{~m}$ y se sitúan a $320 \mathrm{~m}$ y a 490 m en línea recta del área anterior (fig. 3). Además, presentan restos de fosas perimetrales. Éstas estructuras presentan más dudas e interrogantes ya que su morfología puede incluirse en un amplio repertorio de construcciones. A pesar de esta dificultad, creemos que es posible plantear como hipótesis de trabajo su interpretación como torres-vigía similares a las representadas en la Columna de Trajano (Goldsworthy 2003: 154-156).

\section{ENCUADRE DEL CAMPAMENTO EN RELACIÓN AL PROCESO DE IMPLANTACIÓN ROMANA}

Insertar en una línea espacio-temporal los restos localizados en la rivera de Lácara es un ejercicio complicado debido a la falta de una intervención arqueológica sistemática. No obstante, partiendo de la premisa de que estos restos se encuadran cronológicamente en el período romano-republicano debido a que es en esta estapa cuando se producen movimientos militares romanos en esta área peninsular.

Las fuentes documentales nos ofrecen bastante información sobre la implantación del poder romano en el occidente peninsular y, especialmente, sobre su conquista (Martín Bravo 1999: 263). Un proceso, este último, dilatado en el tiempo y definido, en líneas generales, por la resistencia indígena ante el avance de Roma. Sin embargo, la continuidad temporal que podría extraerse de las fuentes clásicas no es una conclusión segura ya que los movimientos de los ejércitos romanos en la península no fueron ni permanentes ni progresivos en el espacio (Morillo 1993). Además, no resulta fácil disertar sobre los avances y retrocesos militares o sobre la identificación de muchos de los recintos militares documentados actualmente, ya que los autores clásicos centraron su atención en aquellos que jugaron un importante papel en la conquista de Hispania o que perduraron más en el tiempo.

Las referencias de autores clásicos a episódicos encuentros entre indígenas y romanos en el occidente peninsular son abundantes y abarcan un amplio territorio, siendo los lusitanos los principales protagonistas. Las fuentes son particularmente insistentes en los primeros conflictos entre lusitanos y romanos, pero tras el "final" de las guerras lusitanas el silencio de los autores clásicos complica el entendimiento de lo que ocurre tras el conflicto. Ello no significa necesariamente que Roma subyugara todo el territorio ni tampoco que no hubiera sublevaciones por parte de los lusitanos. Para algunos autores tras la guerra se estableció una posición de control ubicada en la cuenca hidrográfica del Tajo, donde el campamento de Cáceres el Viejo gana especial relevancia (Fabião 2005: 70). Además, habría que tener en cuenta los sucesos bélicos relacionados con las guerras civiles acaecidas en el último siglo de la República romana, especialmente la Guerra de Sertorio, o las expediciones punitivas de Julio Cesar contra los lusitanos (Novillo 2010). 


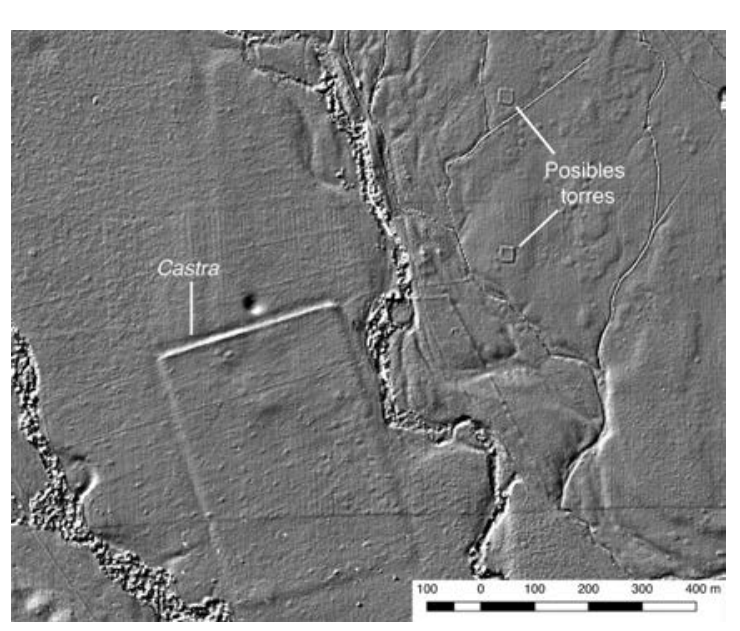

Fig. 3: Estructuras interpretadas en la rivera del Lácara, sombreado analítico del MDT.

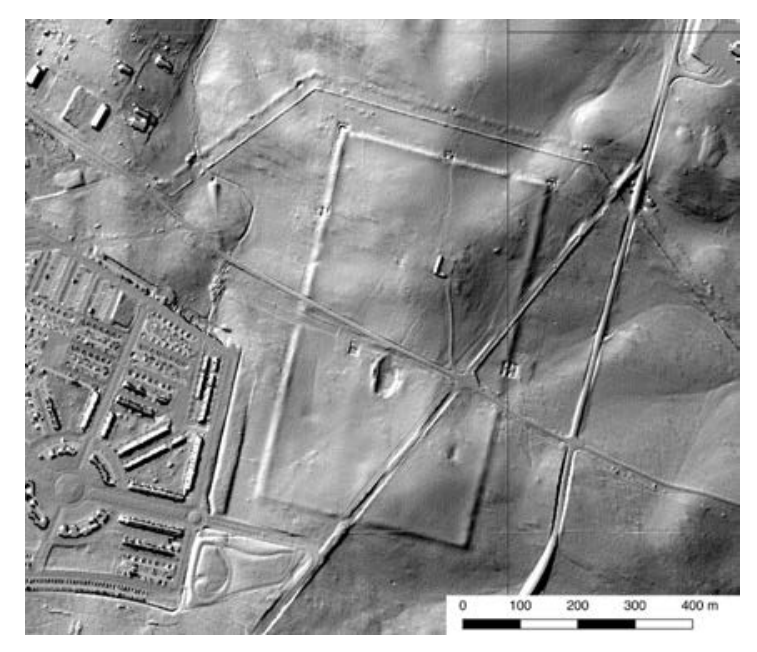

Fig. 4: Campamento romano de Cáceres el Viejo. A diferencia del presentado en este artículo las estructuras que mejor se identifican corresponden a los restos de la muralla.

Los textos clásicos nos permiten conocer el nombre de campamentos que se localizaron en esta área peninsular como Castra Caepiana, Castra Servilia o Castra Caecilia, identificado tradicionalmente, a pesar de que este debate no puede darse por acabado a día de hoy (Abásolo et al. 2008), en el yacimiento de Cáceres el Viejo (Cáceres). De otro lado, en los últimos años, la investigación arqueológica ha permitido aumentar el número de emplazamientos militares romanos en el occidente peninsular. Una investigación que ha sido especialmente dinámica en Portugal, permitiéndonos conocer mejor enclaves militares emblemáticos como Castelo da Lousã (Luz, Mourão) o Lomba do Canho (Secarias, Arganil) (Fabião 2006).

En Extremadura, los estudios de síntesis sobre la ocupación militar romana han sido solapados por el interés generado en el análisis del campamento de Cáceres el Viejo (Abásolo et al. 2008) (fig. 4). Prueba de ello son las diferentes campañas de excavación emprendidas por la Junta de Extremadura en los últimos años, aunque sus resultados permanecen aún inéditos. Si bien en la actualidad las intervenciones arqueológicas sobre enclaves militares romano republicanos en los sitios de El Santo y El Pedrosillo, han renovado el panorama investigador. En el primero, apenas a $30 \mathrm{~km}$ en línea recta del lugar presentado en este trabajo, su excavador identificó diferentes fases sincrónicas de ocupación que se extienden entre los ss. II y I a.n.e., edificándose un perímetro amurallado en el primer cuarto del s. I a.n.e. similar al de Cáceres el Viejo (Heras 2015: 191200). No obstante, su interpretación como recinto militar todavía genera dudas entre investigadores como A. Morillo (2016: 28). Estos titubeos no son extraños dentro del campo de la militaria romana, en el que la realización de intervenciones arqueológicas sobre yacimientos identificados como campamentos legionarios no tienen porqué despejar, necesariamente, las reticencias de la comunidad científica. Este sería, también, el caso de El Pedrosillo. El sitio, datado del s. II a.n.e. y con una extensión estimada de 350 ha, estaría formado por diferentes sistemas defensivos complementarios, destacándose un campamento con una muralla pétrea (sin cimentación y sin foso exterior) que protegería el acuartelamiento en tiendas de la tropa. Además, el lugar estaría cercano a un campo de batalla (Morillo et al.2011). Esta interpretación es puesta en duda por F. J. Heras (2015: 351-352).

El final de las acciones bélicas no dejaría el territorio libre de recintos militares, probablemente algunos continuarían en funcionamiento, aunque con una dotación militar menos numerosa con el objetivo de controlar las comunidades cercanas y evitar posibles sublevaciones. Por otra parte, y suponiendo que los establecimientos con ese fin gozaban de una mayor estabilidad, es posible que fueran de construcción más eficaz y duradera. Sin embargo, es cierto que nos encontramos aún en una fase de conocimiento especulativo en cuanto a la identificación y caracterización de ese tipo de recintos militares (Morillo 2016: 35-37). En este sentido, cabría resaltar la gran dificultad que supone datar y clasificar los recintos militares y, más aún, asociarlos a episodios históricos concretos como 
sucede con un caso paradigmático, y con un dilatado historial de estudios e intervenciones, como es Cáceres el Viejo (Abásolo et al. 2008).

\section{CONSIDERACIONES FINALES}

En el caso del sitio presentado, su definición cronológica, que parece esencial en su integración dentro de la compleja dinámica del proceso de implantación romana en el territorio, deberá ser complementada con la realización de prospecciones y excavaciones arqueológicas que permitan determinar con mayor fiabilidad su contexto histórico.

A pesar de los interrogantes abiertos por la ausencia de trabajos arqueológicos realizados en el sitio, creemos plausible encuadrar el campamento entre los ss. II-I a.n.e., aunque es difícil adscribirlo a un evento histórico concreto, ya que durante estas centurias se suceden en este sector de la península un importante número de acontecimientos militares que debieron originar una considerable actividad constructiva de recintos castrenses. Quizás si nos atenemos a las similitudes formales más básicas con Cáceres el Viejo -esencialmente su plantapodría proponerse una fecha de las primeras décadas del s. I a.n.e., que no deja de ser muy especulativa. Aún así, y en definitiva, no tenemos elementos de juicio suficientes para definir si su presencia en la rivera de Lácara puede relacionarse con el proceso de conquista. De otro lado, en lo que respecta a las dos estructuras de planta cuadrangular localizadas al este del recinto principal, creemos que es posible plantear como hipótesis de trabajo su interpretación como torres-vigía.

\section{BIBLIOGRAFÍA}

ABÁSOLO, J. A.; GONZÁLEZ M. L.; MORA, B. (2008): Recientes investigaciones en el Campamento de Cáceres el Viejo, Arqueología urbana en Cáceres. Investigaciones e intervenciones recientes en la ciudad de Cáceres y su entorno (P. J. Sanabria, ed.), Cáceres, 115-143.

CERRILLO-CUENCA, E. 2017: An approach to the automatic surveying of prehistoric barrows through LiDAR, Quaternary International 435B, 135-145.

DOI: http://doi.org/10.1016/j.quaint.2015.12.099

COSTA-GARCÍA, J. M.; CASAL, R. (2015). Fotografía aérea histórica, satelital moderna y LiDAR aéreo en algunos recintos militares romanos de Castilla y León, Portugalia, $36,143-145$.
COSTA-GARCÍA, J. M.; FONTE, J.; MENÉNDEZ, A.; GONZÁLEZ, D.; GAGO, M.; BLANCO, R.; ÁlVAREZ, V. (2016): Roman Military Settlements in the Northwest of the Iberian Peninsula: The Contribution of Historical and Modern Aerial Photography, Satellite Imagery and Airborne LiDAR, AARGnews. The Newsletter of the Aerial Archaeology Research Group 52, 43-51.

FABIAO, C. (2005): Arqueologia militar romana da Lusitania: textos e evidências materiais, Actas Arqueología Militar Romana en Europa (C. Pérez-González, E. Illarregui, eds.), Salamanca, 55-73.

FABIAO, C. (2006): The Roman Army in Portugal, The Roman Army in Hispania. An Archaeological Guide (A.Morillo, J. Aurrecoechea, eds.), León, 107-126.

GOLDSWORTHY, A. G. (2003): The Complete Roman Army, Londres.

HESSE, R. (2010): LiDAR-derived Local Relief Models-a new tool for archaeological prospection, Archaeological Prospection $17(2), 67-72$. DOI: http://dx.doi.org/10.1002/arp.374

HERAS, F. J. (2015): Arqueología de la implantación romana en los cursos Tajo-Guadiana (siglos II y I a.n.e.), Tesis doctoral, Universidad de Barcelona.

MARTÍN, A. (1999): Los orígenes de la Lusitania: el I Milenio a.C. en la Alta Extremadura, Madrid.

MORILLO, A. (1993): Campamentos romanos en España a través de los textos clásicos, Espacio, Tiempo y Forma. Historia Antigua, serie II, 379-397.

MORILLO, A. (2008): Criterios arqueológicos de identificación de los campamentos romanos en Hispania, Saldvie 8, 93-94.

MORILLO, A. (2016): Campamentos y fortificaciones tardorrepublicanas en Hispania, Fortificaciones y control del territorio en la Hispania republicana (J. Pera, J. Vidal, eds.), Zaragoza, 1-52.

MORILlO, A.; RODRÍGUEZ, F. G.; MARTÍN, E.; DURÁN, R. (2011): The roman republican battlefield at Pedrosillo (Casas de Reina, Badajoz, Spain) new research (2007), Conimbriga 50, 59-78.

DOI: http://dx.doi.org/10.14195/1647-8657_50_3

NOVILLO, M. (2010): La propretura cesariana en la Hispania Ulterior: La II guerra lusitana, Gerión 28, 207-221.

SABUGO, N. (2007): Hispania: huellas de la conquista romana. Aproximación al estudio de los fosos de los asentamientos militares peninsulares, Estudios Humanísticos. Historia 6, 19-46. DOI: http://dx.doi.org/10.18002/ehh.v0i6.3092

ŠTULAR, B.; KOKALI, Ž.; OŠTIR, K.; NUNINGER, L. (2012): Visualization of Lidar-Derived Relief Models for Detection of Archaeological Features, Journal of Archaeological Science 39(11), 3354-3360. DOI: http://doi.org/10.1016/j.jas.2012.05.029

ZHAN, Q.; LIANG, Y.; CAI, Y.; XIAO, Y. (2011): Pattern detection in airborne LiDAR data using Laplacian of Gaussian filter, Geo-spatial Information Sciences 14, 184-189. DOI: http://dx.doi.org/10.1007/s11806-011-0540-x 